\title{
Genetics of photoreceptor degeneration and regeneration in zebrafish
}

\author{
Susan E. Brockerhoff • James M. Fadool
}

Received: 16 August 2010/Revised: 9 September 2010/ Accepted: 5 October 2010/Published online: 24 October 2010

(C) The Author(s) 2010. This article is published with open access at Springerlink.com

\begin{abstract}
Zebrafish are unique in that they provide a useful model system for studying two critically important problems in retinal neurobiology, the mechanisms responsible for triggering photoreceptor cell death and the innate stem cell-mediated regenerative response elicited by this death. In this review we highlight recent seminal findings in these two fields. We first focus on zebrafish as a model for studying photoreceptor degeneration. We summarize the genes currently known to cause photoreceptor degeneration, and we describe the phenotype of a few zebrafish mutants in detail, highlighting the usefulness of this model for studying this process. In the second section, we discuss the several different experimental paradigms that are available to study regeneration in the teleost retina. A model outlining the sequence of gene expression starting from the dedifferentiation of Müller glia to the formation of rod and cone precursors is presented.
\end{abstract}

Keywords Zebrafish · Photoreceptor - Degeneration · Regeneration $\cdot$ Retina

\section{Photoreceptor degeneration}

Retinal photoreceptors are the primary sensory cells responsible for detecting light. There are rods, responsible

\author{
S. E. Brockerhoff $(\bowtie)$ \\ Department of Biochemistry, University of Washington, \\ 357350, Seattle, WA 98195, USA \\ e-mail: sbrocker@uw.edu \\ J. M. Fadool \\ Department of Biological Science, Florida State University, \\ 3018 Life Science Bldg, Tallahassee, FL 32306, USA \\ e-mail: jfadool@bio.fsu.edu
}

for vision at night, and cones, responsible for color and daytime vision. These highly polarized neurons convert light information into an electrical signal that is ultimately transmitted to the vision detecting centers of the brain. The photoreceptor outer segment contains stacks of membranous discs in which the proteins involved in photodetection and transduction reside. The photoreceptor inner segment contains a high density of mitochondria to meet the intense energy demands of the cell, and it is also the site of protein production and sorting. The photoreceptor synapse contains a specialized structure known as a ribbon optimized for continual graded release of neurotransmitter (Fig. 1). The viability and maintenance of these specialized, compartmentalized neurons is critical for normal visual function. Thus, understanding the biology of normal and diseased photoreceptors is of fundamental importance.

The loss of photoreceptors through degeneration results in a devastating incurable form of blindness that represents a major fraction of inherited retinal disease. These disorders can affect either rods or cones individually, as in the case of retinitis pigmentosa or macular degeneration, respectively, or they can cause degeneration of both types of photoreceptors in diseases such as cone-rod dystrophy or Leber's congenital amaurosis. Defects in many different genetic loci are known to cause inherited photoreceptor degeneration, and unique mutations in a single gene can cause different degenerative disorders. These causal genetic loci encode proteins involved in all aspects of photoreceptor biology. For example, mutations in genes for proteins involved in phototransduction, protein transport, energy metabolism, outer segment formation, retinoid metabolism, RNA splicing, and gene transcription have all been identified as causing the various forms of photoreceptor degeneration found in humans (see RETNET for a 


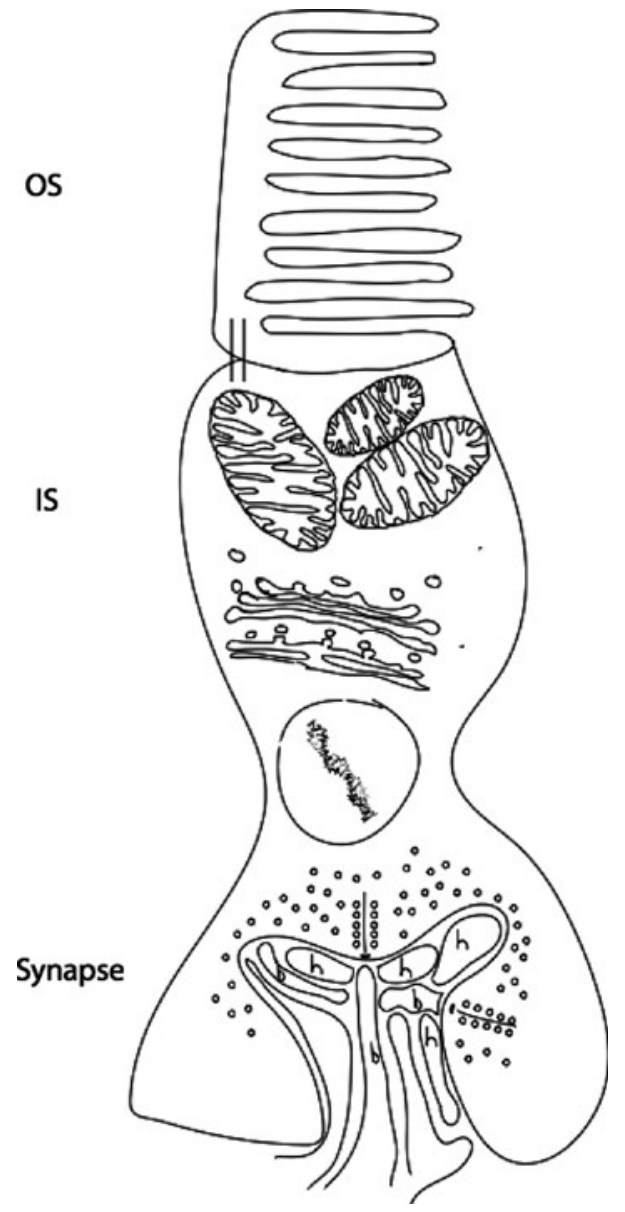

Fig. 1 Schematic of a cone photoreceptor. OS Outer segment, IS inner segment

comprehensive list of retinal disease-causing genes; http://www.sph.uth.tmc.edu/Retnet/).

Zebrafish are considered an outstanding model for studying photoreceptor degenerations. There are several distinct experimental advantages to the zebrafish model system. The ex utero rapid development, optical transparency, permeability to drugs, facile genetics, ease of making transgenics and mosaic animals, as well as the acute visual abilities of larvae, have secured zebrafish as a dominant model organism for studying the visual system. In addition, an exciting new development in recent years has been the identification of many gene mutations isolated from fish with photoreceptor degeneration phenotypes (Table 1). More than a dozen different genetic lesions causing photoreceptor degeneration are now known. These fish were identified in genetic screens that focused on identifying visual behavioral or morphology deficits, and subsequent cloning strategies have led to the identification of the mutated genes. The list provided in Table 1 presents the mutant name, causal gene, gene function and corresponding references. Importantly, this list only includes mutants with known genetic mutations. The relevance of the zebrafish as a powerful model is further highlighted by the multiple categories of genes represented in this list. Proteins essential for phototransduction, protein/organelle transport, metabolism, and transcription have been isolated. Thus the molecular mechanisms triggering cell death due to unique primary lesions can be investigated using these zebrafish mutants. This provides a powerful scientific resource since the cascade of molecular events leading to cell death has not yet been determined in any organism even for a single mutation that causes photoreceptor degeneration.

To date, most of the photoreceptor degeneration mutants die within 2 weeks post-fertilization. This is not surprising since the majority of cloned mutants were isolated based on morphological criteria (Table 1). This indicates that these genes have multiple functions within the organism, and for some mutations the photoreceptor phenotype is at least partially a secondary consequence of defects in other cells. For example, in dynactin 1 and 2 mutants (mok and ako), the photoreceptor phenotype contains both cell-autonomous and non-cell-autonomous components [1-3]. Similarly, in rep 1 mutants, photoreceptor degeneration is caused by the presence of neighboring mutant retinal pigment epithelial cells [4]. In contrast, mutations isolated solely on the basis of visual behavioral defects are less likely to have pleiotropic effects. pde6c represents a gene in this category, and fish carrying mutations in this gene are viable as adults [5, 6]. Additional adult viable mutants with photoreceptor deficiencies were isolated in a large-scale behavioral screen [7], however, the genetic lesion responsible for the photoreceptor loss in these fish has not been determined. Behavioral screening strategies still represent the most efficient and likely successful strategy for identifying photoreceptorspecific lesions. The currently small number of behavioral mutations - the majority of which are single allele-indicates that many more mutations specifically affecting photoreceptor viability will be isolated in the future.

Many of the experimental advantages of the zebrafish for studying retinal degenerations are highlighted in a recent study using a cone-specific degeneration model with a mutation in the $p d e 6 c$ gene [8]. pde6c encodes a conespecific version of phosphodiesterase essential for phototransduction in cone photoreceptors. A complete loss of Pde6c leads to the death of cone photoreceptors during the first week of zebrafish development [5]. An analogous mouse mutant containing a mutation in the rod-specific version of cGMP-phosphodiesterase has been available for decades, and yet the mechanism triggering apoptosis of photoreceptors due to phosphodiesterase deficiency is still unknown [9]. Using transgenic zebrafish expressing fluorescent reporter proteins in cones, photoreceptor cell death was visualized in real time in live pde6c mutant animals, and the sequence of morphological changes occurring 
Table 1 Zebrafish photoreceptor degeneration mutants

\begin{tabular}{|c|c|c|c|c|}
\hline $\begin{array}{l}\text { Mutant name/ } \\
\text { allele }\end{array}$ & Causal gene & Protein function & Phenotype & $\begin{array}{l}\text { Gene/ } \\
\text { mutation } \\
\text { identification }\end{array}$ \\
\hline$p o b^{a l}$ & Partial optokinetic response b; GeneID: 406826 & Unknown & $\begin{array}{l}\text { Red cone degeneration } \\
\quad 4 \mathrm{dpf}\end{array}$ & [74] \\
\hline $\begin{array}{l}\text { pde6c } c^{w 59} \\
e l s^{r w 76 a}\end{array}$ & Phosphodiesterase 6c; GeneID: 5146 & $\begin{array}{l}\text { Hydrolysis of cGMP during } \\
\text { phototransduction }\end{array}$ & $\begin{array}{l}\text { Cone degeneration } 4 \mathrm{dpf} \\
\text { onward }\end{array}$ & {$[5,6]$} \\
\hline$o v l^{t z 288 b}$ & Intraflagellar transport (Ift) 88; GeneID: 321855 & Intraflagellar transport & $\begin{array}{l}\text { Photoreceptor } \\
\text { degeneration } 4 \mathrm{dpf}\end{array}$ & {$[18]$} \\
\hline$n r f 1^{h i 39 a}$ & Nuclear respiratory factor 1; GeneID: 64604 & Transcription factor & $\begin{array}{l}\text { Photoreceptor } \\
\quad \text { degeneration } 3 \mathrm{dpf}\end{array}$ & {$[75]$} \\
\hline$p w i^{h i 1482}$ & Tryptophan rich basic protein; GeneID: 335756 & Unknown & $\begin{array}{l}\text { Photoreceptor } \\
\quad \text { degeneration } 2-3 \mathrm{dpf}\end{array}$ & {$[19]$} \\
\hline ift172 $2^{h i 2211}$ & Ift172; GeneID: 432389 & Intraflagellar transport & $\begin{array}{l}\text { Photoreceptor } \\
\quad \text { degeneration } 4 \mathrm{dpf}\end{array}$ & [19] \\
\hline ift $57^{h i 3417}$ & Ift57; GeneID: 414929 & Intraflagellar transport & $\begin{array}{l}\text { Photoreceptor } \\
\text { degeneration } 4 \mathrm{dpf}\end{array}$ & [19] \\
\hline $\begin{array}{l}\text { elipsa }^{m 649} \\
\quad \text { elipsa }^{t p 49 d}\end{array}$ & $\begin{array}{l}\text { TNF receptor-associated factor } 3 \text { interacting } \\
\text { protein 1; GeneID: } 393572\end{array}$ & Component of IFT particle B & $\begin{array}{l}\text { Photoreceptor } \\
\quad \text { degeneration } 4 \mathrm{dpf}\end{array}$ & {$[21]$} \\
\hline$a k o^{j j 50}$ & Dynactin 2 (dynamitin); GeneID: 394141 & Intracellular organelle transport & $\begin{array}{l}\text { Photoreceptor } \\
\text { degeneration } 3 \mathrm{dpf}\end{array}$ & [2] \\
\hline $\begin{array}{l}m o k^{s 309} \\
m^{2} k^{m 632}\end{array}$ & Dynactin 1a (p150); GeneID: 407638 & Intracellular organelle transport & $\begin{array}{l}\text { Photoreceptor } \\
\quad \text { degeneration } 3 \mathrm{dpf}\end{array}$ & {$[1,76]$} \\
\hline$f l r^{m 477}$ & Fleer, TPR repeat protein 30; GeneID: 797829 & Tubulin polyglutamylation & $\begin{array}{l}\text { Photoreceptor } \\
\quad \text { degeneration } 4 \mathrm{dpf}\end{array}$ & {$[20]$} \\
\hline $\begin{array}{l}s y u^{t 4+/-} \\
s y u^{t b x+/-}\end{array}$ & Shha, sonic hedgehog a; GeneID: 30269 & $\begin{array}{l}\text { Maintenance/survival of the cones } \\
\text { in the adult retina }\end{array}$ & Slow adult degeneration & {$[23]$} \\
\hline repl $1^{\text {rus48 }}$ & Rab escort protein-1; GeneID: 402,935 & Directs rab protein movement & $\begin{array}{l}\text { Photoreceptor } \\
\quad \text { degeneration } 4 \mathrm{dpf}\end{array}$ & [77] \\
\hline
\end{tabular}

$d p f$ days post fertilization

during this process was categorized. Using two-photon microscopy, the authors found that the first sign of death was a retraction of synaptic processes within the outer plexiform layer. This was followed by shrinkage and collapse of the outer segment. These morphological changes provide the foundation for examining subcellular changes in fluorescent signaling molecules as photoreceptors are undergoing apoptosis.

The authors further exploit the ease of generating mosaic fish to examine whether mutant cone photoreceptors trigger neighboring wild-type (WT) cone death in a process known as bystander-associated cell death [8]. A well-known example of this is in the human disease retinitis pigmentosa (RP), where dying rods cause the secondary death of healthy cones [10]. The molecular mechanism causing the secondary death of healthy photoreceptors is unknown, and thus animal models are useful for studying this process [11]. For example, the underlying mechanism of cone death in rod dystrophies is actively being studied in the mouse [12]. Analysis in humans indicates that the extent of bystander cell death is not the same for all classes of retinal degeneration [13-15]. In addition, the ability of dying cones to affect neighboring healthy cones is unknown. The cone dominance of the larval zebrafish retina made it possible using this model system to evaluate whether dying cones could cause the secondary death of neighboring healthy cones [8]. By generating zebrafish with heterogenous retinas containing mixtures of WT and pde6c mutant cones, parameters of transplant size and position were carefully evaluated for their influence on bystander-associated cone death. Surprisingly, although dying mutant cones could cause rod death in certain retinal regions [5], mutant cones did not adversely affect the viability of WT cones regardless of transplant size or position within the retina. This finding suggests that cones may be more likely to remain viable in transplant experiments aimed at rescuing vision in degenerating retinas. These findings with the pde6c mutant also suggest that this model may be useful for studying the ability of dying cones to cause neighboring WT rod death [5]. However, in contrast to the mammalian system, the ability of degenerating rods to cause neighboring WT cone death has not been observed in the zebrafish with the degeneration models that are currently available [16]. 
To date, the majority of mutants with photoreceptor degeneration have mutations in proteins critical for ciliary transport from the inner to the outer segment (ovl, $f t r$, ift57, ift172, and elipsa). The process of ciliary transport is complicated, involving multiple large transport complexes and regulators, and this process is currently being actively studied by many investigators using a variety of model systems. A recent review nicely summarizes the current status of this field as it pertains to photoreceptor morphogenesis [17]. The zebrafish is contributing to this field through the identification of several essential components and through an analysis of genetic interactions between components.

The first cloned zebrafish mutation essential for ciliary transport of proteins to the outer segment was ovl, a gene encoding one of the intraflagellar transport proteins, IFT88 [18]. A subsequent rescreen of insertional mutants identified additional IFT components, IFT57 and IFT172, as causing photoreceptor degeneration [19]. Two other factors identified are the fleer gene, a modulator of tubulin posttranslational modification [20], and most recently Elipsa [21], a polypeptide thought to link the IFT complex polypeptide IFT20 with Rabaptin 5, an endocytosis regulator. The ability to quickly analyze gene interactions using transient gene knockdown experiments has also been instrumental in connecting elipsa to rabaptin 5 in ciliary transport [21]. All five of these mutants show deficiencies in outer segment formation accompanied by photoreceptor degeneration, and although the mutants have multiple defects, fleer, elipsa, and ovl are known to function cell autonomously in causing photoreceptor degeneration [22]. It is likely that IFT57 and IFT172 have cell autonomous photoreceptor functions as well. The abundance of mutations in this category and the many protein molecules involved in ciliary transport suggest that several additional genes in this pathway will be isolated using the zebrafish model system.

In addition to early-onset rapid degeneration, zebrafish offer the potential to study slower and late-onset degeneration. One study has evaluated photoreceptor abnormalities associated with postreproductive senescence due to suboptimal levels of sonic hedgehog expression [23]. Sonic hedgehog (shh) is known to play an important role in zebrafish retinal development. shh regulates neurogenesis during embryonic development [24], and later, it regulates differentiation of retinal neurons, including photoreceptor cells [25]. In contrast, the role of shh in the adult retina, where it is also expressed, is unknown. Although lethal as a homozygous mutation, fish heterozygous for mutations in the sonic hedgehog gene survive to adulthood. By taking advantage of chronically reduced shh expression in heterozygous carriers, the investigators evaluated the effect of long-term shh deprivation [23]. Interestingly, by 2 years of age dramatic disruptions in photoreceptor patterning and reductions in photoreceptor number were apparent in the heterozygous fish but not in control fish. These disruptions were detected with markers specific to blue, red or green cones. These studies highlighted both the continued role for sonic hedgehog in adult photoreceptor maintenance/ renewal and the usefulness of zebrafish for studying degeneration in older fish.

Another strategy that is just starting to be exploited is the targeted expression of photoreceptor-specific genes in order to elicit degeneration. The identification of promoters to express genes specifically in rod photoreceptors [26], all cones [27], and cone subtypes [28, 29] makes these studies imminent. As an initial example of this type of study, the rod photoreceptor-specific promoter was used to derive expression of membrane-tagged cyan fluorescent protein to rod photoreceptors resulting in their apoptosis [16]. The combined use of photoreceptor-specific promoters with other knockout and gene expression strategies, reviewed in detail elsewhere [30-35], will provide additional experimental paradigms for studying the mechanisms of photoreceptor degeneration in both young and older retinas.

In summary, at least 13 mutations causing photoreceptor degeneration in zebrafish are now known. Many additional mutations causing degeneration will be identified through genetic screens and targeted gene knockout approaches. Transgenic approaches will enable investigators to examine hereditary eye disorders due to known mutations in photoreceptor specific genes. It is likely that zebrafish retinal disease models will continue to contribute significant new findings to the field of photoreceptor degeneration.

\section{Regeneration}

During the past decade, there has been tremendous resurgence in interest from both the scientific community and the general public in neural stem/progenitor cells and the prospect of novel therapies for trauma-induced injury and degenerative diseases of the retina. Several independent yet related lines of evidence have contributed to the optimistic outlook: the demonstration of continuous neurogenesis in discrete regions of the vertebrate retina, the ability to isolate and expand in culture cells with stem cell-like properties, and the demonstration that Müller glia can divide and give rise to neuroprogenitors [36-38]. In teleosts including zebrafish, mechanical damage, chemical toxicity, phototoxicity, and genetic lesions stimulated an increase in proliferation of cells in the inner nuclear layer (INL) and outer nuclear layer (ONL), regeneration of the damaged photoreceptors [39-42], and recovery of vision [43]. 
The regeneration of the retina shares features common to other regenerating tissues including the heart and fin [44]. To translate the recent advances into clinical treatments, three basic questions need to be answered more fully: What are the intrinsic properties of retinal stem cells? How do environmental signals direct regeneration of the photoreceptor cells? Can the intrinsic and extrinsic properties be manipulated to direct photoreceptor cell replacement? The regeneration of rod and cone photoreceptors in the teleost provides a model to systematically investigate the potential and the limitations of neural stem cells for replacement of lost photoreceptor cells.

Persistent neurogenesis and regeneration in the visual system of adult teleost fish have been valuable models of neural development. Zebrafish, like many teleosts, continue to grow throughout their life, and the increase in body mass is matched by an increase in the size of the eye and the area of the retina [45-49]. As the fish grows, new neurons, including photoreceptor cells, are added at the retinal margin by a population of mitotic progenitor cells located within the circumferential mitotic zone. The mitotic progenitors possess properties of stem cells, maintaining a balance between self-renewal and the generation of multipotent neuroblasts that differentiate into all classes of retinal neurons and glia. The temporal pattern of expression of transcription factors and signaling molecules in the retinal margin recapitulates a developmental program. The isolation of proliferative cells from the ciliary margin of rodents and identification of persistent, but limited neurogenesis at the margin of the hatchling chick retina suggest a conserved system, although further experimentation is warranted [36, 50-53].

Perhaps more relevant to this review is that, during postembryonic growth in teleosts, new rods are generated in the central retina from a second population of mitotic cells referred to as the rod progenitor lineage. As the retina is gradually stretched within the expanding optic cup, the addition of new rods maintains their constant density across the ONL, preserving scotopic sensitivity [45, 46, 49, 54]. Proliferating cells distributed across the ONL, termed rod precursors, were initially identified as the source of the newly generated rods [46, 47]. The rod precursors slowly divide followed by differentiation as rod photoreceptors. Subsequent studies using multiple injections of tritiated thymidine or exposure to thymidine analogues revealed groups of mitotically active cells arranged in radial arrays spanning the INL and ONL. In histological sections, these neurogenic clusters appeared to migrate along Müller glia. The authors proposed that the clusters of proliferating cells migrated to the outer retina and became the source of the rod precursors. The generation of functional photoreceptors from a population of mitotic precursors is precisely the goal of stem cell replacement therapy.
Several different models identified a subpopulation of Müller glia as the origin of both the rod progenitor lineage and the "neurogenic clusters" observed in regenerating fish retinas [55-61]. Müller glia-derived proliferating cells possess the capacity to regenerate virtually all classes of retinal neurons in teleosts. In the chick, the capacity of Müller glia regeneration is limited to small numbers of inner retinal neurons, and in the mouse, Müller glia proliferation and regeneration is even more severely limited [37, 38, 62, 63]. Double labeling experiments in zebrafish demonstrated that these proliferating cells colabel for definitive markers of Müller cells including glial fibrillary acid protein (GFAP), carbonic anhydrase, and glutamine synthetase. Barnados et al. [55, 64] used transgenic zebrafish in which regulatory elements of the zebrafish GFAP gene were used to drive expression of cytoplasmic or nuclear-targeted GFP specifically in Müller glia. The authors used the persistence of GFP fluorescence as a lineage tracer of cells derived from the Müller glia. Following BrdU labeling to identify mitotically active cells in the uninjured retina, the authors observed a small number of BrdU+ cells in the INL and ONL and some of those were GFP+. Following light damage, the number of mitotically active GFP+ cells increased considerably and could be traced as clusters extending from the INL to the ONL, where some expressed markers of differentiating photoreceptors. The authors concluded that the colabeled cells were dedifferentiated Müller glia and their progeny that migrate from the INL into the ONL, forming rod precursors and regenerated photoreceptors, respectively. It has also been demonstrated that constant intense light treatment of dark-adapted albino zebrafish selectively kills rod and cone photoreceptors in the central retina [41, 44], and induces approximately $50 \%$ of the Müller glia to colabel for mitotic markers such as PCNA [65]. Injection and electroporation of antisense morpholinos complementary to PCNA prior to retinal lesion led to a significant increase in the number of dying cells in the INL and reduced both the number of proliferating cells and the number of Müller glia in the region of the light damage [65]. These data suggest that, following retinal lesion, asymmetric cell division of Müller glia generates a mitotic progenitor that gives rise to the neurogenic cluster while maintaining a constant population of Müller glia.

The Müller glia-derived mitotic cells appear to initiate expression of retinal stem/progenitor cell markers, including Ascl1a, Pax6, Rx1, Neurogenin1, and Chx10 [40, 66, 67]. Ash1a, a basic helix-loop-helix transcription factor and regulator of vertebrate neurogenesis, is expressed within $4 \mathrm{~h}$ post lesion, making it one of the earliest markers of the proliferating Müller glia. Knockdown of Asclla results in a reduction in divided cells following retinal lesions and inhibits regeneration, but unlike PCNA 
knockdown, interfering with Ascl1a expression did not lead to cell death [40]. These data would suggest that activation of Asclla is an early step in the reprogramming of quiescent Müller glia. A putative downstream target of Ascl1a is Pax6, a paired box transcription factor with highly conserved roles in retinal and ocular development in species as diverse as flies and mammals. Previous studies in goldfish have shown that, in addition to expression by a subpopulation of inner retinal neurons in the mature retina, Pax6 is expressed in a rare population of cells within the INL thought to be the source of the mitotic progenitors [68]. Following surgical removal of a region of the retina or after light lesion, Pax6 immunoreactivity colocalized with $\mathrm{BrdU}$ in the regenerating tissue. As a vestige of the ancestral duplication of the teleost genome, zebrafish retained two Pax6 co-orthologues, one encoded by the pax6a gene and the other encoded by the pax6b gene [67]. Knockdown of either pax6a or pax $6 b$ by intravitreal injection and electroporation of morpholinos that were complementary to either mRNA revealed subfunctionalization of the corresponding proteins during photoreceptor regeneration [67]. Loss of Pax6b expression did not affect Müller glial cell division but was required for subsequent division of the neuronal progenitors. Inhibition of the Pax6a protein blocked subsequent neuronal progenitor cell divisions and decreased the number of cells in the neurogenic cluster. Significantly fewer cones were identified in the pax $6 b$ and pax6alpax6b double morphant retinas suggesting that INL proliferation is necessary for cone regeneration [67]. Based upon these and other results, a model of the sequence of gene expression leading from the activation of the Müller glia to the formation of photoreceptor precursors can be drawn (Fig. 2).

As regeneration progresses, the Müller-derived stem cells expressed numerous genes consistent with a photoreceptor developmental program. As the retinal progenitors migrate from the INL to the ONL, pax6 expression is lost and other transcription factors are expressed, most notably the basic helix-loop-helix gene neuroD and the cone-rod homeobox gene $\operatorname{crx}$. Similarly, cell signaling molecules of the Notch-Delta pathway and its downstream effectors are also expressed [66]. These data suggest that progenitor cells produced in the INL in response to acute injury pass through a series of intervening, less-committed states prior to adopting a photoreceptor cell fate. Ultimately, the gliaderived cells exit the cell cycle to differentiate as photoreceptors that integrate into the existing retinal circuitry and restore vision [43].

Whereas current work in numerous species has focused on the Müller cells and the INL stem cells, understanding the regulation of rod progenitor cell activity may offer an alternative avenue to investigate the fundamental processes of photoreceptor replacement. Two previous limitations of

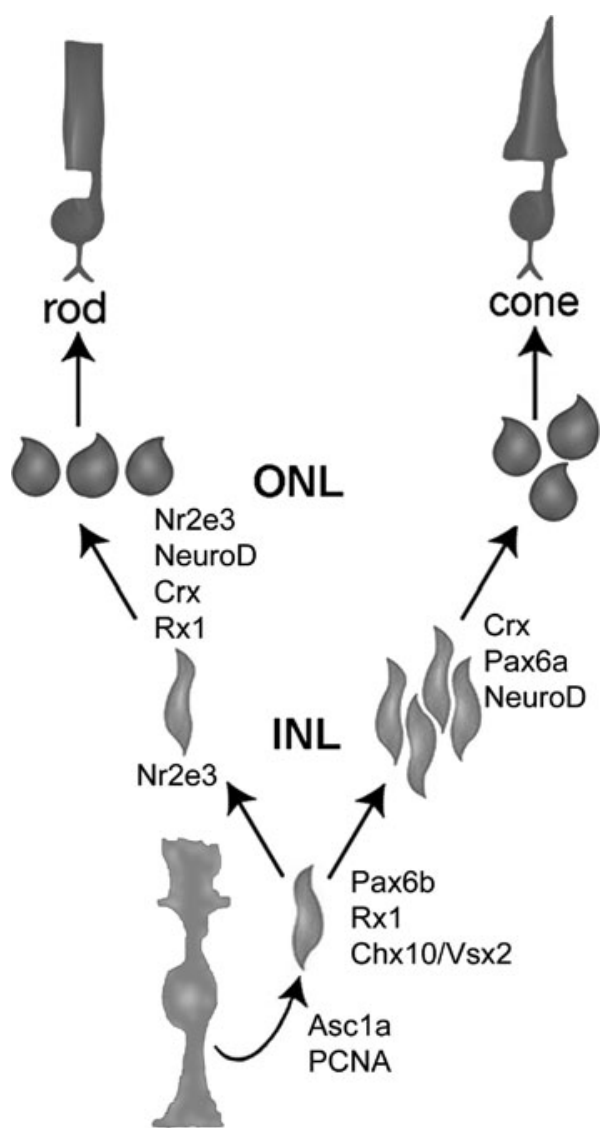

Fig. 2 A model for gene expression during persistent and injury- or degeneration-induced neurogenesis in the zebrafish central retina. Adapted from [58], see text for details. ONL Outer nuclear layer, INL inner nuclear layer

such studies have been that rod precursors are relatively few in number and no definitive marker is available. The generation of a transgenic line of zebrafish, the XOPSmCFP line, which experiences selective degeneration of the rod photoreceptor cells due to the toxic effect of a rodtargeted fluorescent reporter gene [16], facilitates new analyses. This rod degeneration resulted in a loss of rodmediated electrophysiological responses but did not cause any secondary cone pathology. It was, however, accompanied by a significant increase in rod progenitor mitotic activity [16]. Similar to cone regeneration, the transcription factors NeuroD and Crx are upregulated in cells in the ONL. But in contrast, expression of $n r 2 e 3$, a rod determination gene that is expressed in mammals exclusively in postmitotic differentiating rod photoreceptors, was also upregulated, whereas expression of pax6 was not observed [16]. Immunolabeling of retinal cryosections with antiBrdU showed no significant increase in INL BrdU+ cells or PCNA expression. The expression of genes with demonstrated roles in photoreceptor development, neuroD, crx, and $n r 2 e 3$, and lack of expression of pax6 suggests an early commitment of the mitotic rod precursors to the rod cell 
fate [16]. Supporting this conclusion, knockdown of both pax6a and pax $6 b$ resulted in increased numbers of rod precursors in the ONL and rod regeneration continued [67]. Therefore, it appears that the rod progenitors in the ONL maintain the capacity to respond to rod photoreceptor degeneration without relying on increased activity of progenitor cells in the INL or the Müller glia. These results are particularly interesting in light of a report that demonstrated that in mouse both mitotic retinal progenitors and postmitotic rod precursors could differentiate into rhodopsin-expressing cells upon transplantation into a mouse model of retinal degeneration, but only the postmitotic Nrlpositive cells could migrate to the appropriate position and integrate correctly into the host retina [69].

Recent work has begun to identify the signaling that regulates the neurogenic program in Müller glia and their derivatives in situ. Previous studies have implicated the growth hormone/insulin-like growth hormone I axis as a regulator of growth and mitotic activity of progenitors in the retina of goldfish, which may be more tightly associated with integrating numerous general features of overall growth and body size [70]. Phagocytosis of cellular debris stimulates mitotic activity and the regenerative potential of Müller glia in the light-damaged retina [71]. Based upon expression patterns following retinal lesion or from the perspective of a candidate gene approach, various molecules have been linked to specific aspects of the regenerative process. More recently, the zebrafish galectin, Drgal1-L2, has been shown to be expressed by Müller glia and neurogenic clusters [72]. Functional studies demonstrate that knockdown led to a decrease in the regeneration of rod photoreceptors but did not affect cone regeneration. Based upon studies in mammals, a neuroprotective effect of ciliary neurotrophic factor (CNTF) upon the lightlesioned zebrafish retina was observed [73]. Unexpectedly, intraocular injection of CNTF into undamaged eyes mimicked the increase in the number of proliferating Müller glia and neuronal progenitors typically observed following retinal lesions. The increase in mitotic activity was preceded by expression of Stat3, and knockdown of Stat3 expression in the CNTF-injected retinas significantly reduced the number of proliferating Müller glia [73]. Though these studies are only beginning, the results suggest an optimistic outlook for identifying compounds that stimulate resident cells to initiate a neurogenic program as an alternative strategy to stem cell replacement therapies.

In summary, investigations using the zebrafish have unequivocally identified Müller glia as a source of the regenerative cells in the neural retina and genes involved in the regenerative process. The existing genetic mutations leading to photoreceptor cell dystrophies and the isolation of new lesions as models of specific heritable disorders will be invaluable research tools to continue to unravel the molecular and cellular pathways regulating the regenerative process. Yet, while significant progress has been made, new approaches are needed to translate these findings to specifically induce the proliferation of resident cells in the mammalian retina, engineer their fates, and direct their incorporation into an existing retinal circuit.

Acknowledgments This article was financially supported by NIH grants EY018814(SEB) and EY017753(JMF).

Open Access This article is distributed under the terms of the Creative Commons Attribution Noncommercial License which permits any noncommercial use, distribution, and reproduction in any medium, provided the original author(s) and source are credited.

\section{References}

1. Tsujikawa M, Omori Y, Biyanwila J, Malicki J (2007) Mechanism of positioning the cell nucleus in vertebrate photoreceptors. Proc Natl Acad Sci USA 104:14819-14824

2. Jing X, Malicki J (2009) Zebrafish ale oko, an essential determinant of sensory neuron survival and the polarity of retinal radial glia, encodes the p50 subunit of dynactin. Development 136:2955-2964

3. Doerre G, Malicki J (2001) A mutation of early photoreceptor development, mikre oko, reveals cell-cell interactions involved in the survival and differentiation of zebrafish photoreceptors. J Neurosci 21:6745-6757

4. Krock BL, Bilotta J, Perkins BD (2007) Noncell-autonomous photoreceptor degeneration in a zebrafish model of choroideremia. Proc Natl Acad Sci USA 104:4600-4605

5. Stearns G, Evangelista M, Fadool JM, Brockerhoff SE (2007) A mutation in the cone-specific pde6 gene causes rapid cone photoreceptor degeneration in zebrafish. J Neurosci 27:13866-13874

6. Nishiwaki Y, Komori A, Sagara H, Suzuki E, Manabe T, Hosoya T, Nojima Y, Wada H, Tanaka H, Okamoto H, Masai I (2008) Mutation of cGMP phosphodiesterase 6alpha'-subunit gene causes progressive degeneration of cone photoreceptors in zebrafish. Mech Dev 125:932-946

7. Muto A, Orger MB, Wehman AM, Smear MC, Kay JN, PageMcCaw PS, Gahtan E, Xiao T, Nevin LM, Gosse NJ, Staub W, Finger-Baier K, Baier H (2005) Forward genetic analysis of visual behavior in zebrafish. PLoS Genet 1:e66

8. Lewis A, Williams P, Lawrence O, Wong RO, Brockerhoff SE (2010) Wild-type cone photoreceptors persist despite neighboring mutant cone degeneration. J Neurosci 30:382-389

9. Sancho-Pelluz J, Arango-Gonzalez B, Kustermann S, Romero FJ, van Veen T, Zrenner E, Ekstrom P, Paquet-Durand F (2008) Photoreceptor cell death mechanisms in inherited retinal degeneration. Mol Neurobiol 38:253-269

10. Hamel C (2006) Retinitis pigmentosa. Orphanet J Rare Dis 1:40

11. Krebs MP, White DA, Kaushal S (2009) Biphasic photoreceptor degeneration induced by light in a T17 M rhodopsin mouse model of cone bystander damage. Invest Ophthalmol Vis Sci 50:2956-2965

12. Punzo C, Kornacker K, Cepko CL (2009) Stimulation of the insulin/mTOR pathway delays cone death in a mouse model of retinitis pigmentosa. Nat Neurosci 12:44-52

13. Michaelides M, Hunt DM, Moore AT (2004) The cone dysfunction syndromes. Br J Ophthalmol 88:291-297

14. Hamel CP (2007) Cone rod dystrophies. Orphanet J Rare Dis 2:7 
15. Cideciyan AV, Hood DC, Huang Y, Banin E, Li ZY, Stone EM, Milam AH, Jacobson SG (1998) Disease sequence from mutant rhodopsin allele to rod and cone photoreceptor degeneration in man. Proc Natl Acad Sci USA 95:7103-7108

16. Morris AC, Schroeter EH, Bilotta J, Wong RO, Fadool JM (2005) Cone survival despite rod degeneration in XOPS-mCFP transgenic zebrafish. Invest Ophthalmol Vis Sci 46:4762-4771

17. Kennedy B, Malicki J (2009) What drives cell morphogenesis: a look inside the vertebrate photoreceptor. Dev Dyn 238:2115-2138

18. Tsujikawa M, Malicki J (2004) Intraflagellar transport genes are essential for differentiation and survival of vertebrate sensory neurons. Neuron 42:703-716

19. Gross JM, Perkins BD, Amsterdam A, Egana A, Darland T, Matsui JI, Sciascia S, Hopkins N, Dowling JE (2005) Identification of zebrafish insertional mutants with defects in visual system development and function. Genetics 170:245-261

20. Pathak N, Obara T, Mangos S, Liu Y, Drummond IA (2007) The zebrafish fleer gene encodes an essential regulator of cilia tubulin polyglutamylation. Mol Biol Cell 18:4353-4364

21. Omori Y, Zhao C, Saras A, Mukhopadhyay S, Kim W, Furukawa T, Sengupta P, Veraksa A, Malicki J (2008) Elipsa is an early determinant of ciliogenesis that links the IFT particle to membrane-associated small GTPase Rab8. Nat Cell Biol 10:437-444

22. Doerre G, Malicki J (2002) Genetic analysis of photoreceptor cell development in the zebrafish retina. Mech Dev 110:125-138

23. Stenkamp DL, Satterfield R, Muhunthan K, Sherpa T, Vihtelic TS, Cameron DA (2008) Age-related cone abnormalities in zebrafish with genetic lesions in sonic hedgehog. Invest Ophthalmol Vis Sci 49:4631-4640

24. Masai I, Yamaguchi M, Tonou-Fujimori N, Komori A, Okamoto H (2005) The hedgehog-PKA pathway regulates two distinct steps of the differentiation of retinal ganglion cells: the cell-cycle exit of retinoblasts and their neuronal maturation. Development 132:1539-1553

25. Stenkamp DL, Frey RA, Prabhudesai SN, Raymond PA (2000) Function for Hedgehog genes in zebrafish retinal development. Dev Biol 220:238-252

26. Fadool JM (2003) Development of a rod photoreceptor mosaic revealed in transgenic zebrafish. Dev Biol 258:277-290

27. Kennedy BN, Alvarez Y, Brockerhoff SE, Stearns GW, SapettoRebow B, Taylor MR, Hurley JB (2007) Identification of a zebrafish cone photoreceptor-specific promoter and genetic rescue of achromatopsia in the nof mutant. Invest Ophthalmol Vis Sci 48:522-529

28. Luo W, Williams J, Smallwood PM, Touchman JW, Roman LM, Nathans J (2004) Proximal and distal sequences control UV cone pigment gene expression in transgenic zebrafish. J Biol Chem 279:19286-19293

29. Takechi M, Seno S, Kawamura S (2008) Identification of cisacting elements repressing blue opsin expression in zebrafish UV cones and pineal cells. J Biol Chem 283:31625-31632

30. Moens CB, Donn TM, Wolf-Saxon ER, Ma TP (2008) Reverse genetics in zebrafish by TILLING. Brief Funct Genomic Proteomic 7:454-459

31. Hans S, Kaslin J, Freudenreich D, Brand M (2009) Temporallycontrolled site-specific recombination in zebrafish. PLoS One 4:e4640

32. Amacher SL (2008) Emerging gene knockout technology in zebrafish: zinc-finger nucleases. Brief Funct Genomic Proteomic 7:460-464

33. Wong AC, Draper BW, Van Eenennaam AL (2010) FLPe functions in zebrafish embryos. Transgenic Res. doi:10.1007/s11248010-9410-9

34. Collins RT, Linker C, Lewis J (2010) MAZe: a tool for mosaic analysis of gene function in zebrafish. Nat Methods 7:219-223
35. Esengil H, Chen JK (2008) Gene regulation technologies in zebrafish. Mol Biosyst 4:300-308

36. Ahmad I (2001) Stem cells: new opportunities to treat eye diseases. Invest Ophthalmol Vis Sci 42:2743-2748

37. Fischer AJ, McGuire CR, Dierks BD, Reh TA (2002) Insulin and fibroblast growth factor 2 activate a neurogenic program in Muller glia of the chicken retina. J Neurosci 22:9387-9398

38. Fischer AJ, Reh TA (2001) Muller glia are a potential source of neural regeneration in the postnatal chicken retina. Nat Neurosci $4: 247-252$

39. Braisted JE, Essman TF, Raymond PA (1994) Selective regeneration of photoreceptors in goldfish retina. Development 120:2409-2419

40. Fausett BV, Goldman D (2006) A role for alpha1 tubulinexpressing Muller glia in regeneration of the injured zebrafish retina. J Neurosci 26:6303-6313

41. Vihtelic TS, Hyde DR (2000) Light-induced rod and cone cell death and regeneration in the adult albino zebrafish (Danio rerio) retina. J Neurobiol 44:289-307

42. Wu DM, Schneiderman T, Burgett J, Gokhale P, Barthel L, Raymond PA (2001) Cones regenerate from retinal stem cells sequestered in the inner nuclear layer of adult goldfish retina. Invest Ophthalmol Vis Sci 42:2115-2124

43. Mensinger AF, Powers MK (1999) Visual function in regenerating teleost retina following cytotoxic lesioning. Vis Neurosci $16: 241-251$

44. Qin Z, Barthel LK, Raymond PA (2009) Genetic evidence for shared mechanisms of epimorphic regeneration in zebrafish. Proc Natl Acad Sci USA 106:9310-9315

45. Fernald RD (1990) Teleost vision: seeing while growing. J Exp Zool Suppl 5:167-180

46. Johns PR (1982) Formation of photoreceptors in larval and adult goldfish. J Neurosci 2:178-198

47. Johns PR, Fernald RD (1981) Genesis of rods in teleost fish retina. Nature 293:141-142

48. Marcus RC, Delaney CL, Easter SS Jr (1999) Neurogenesis in the visual system of embryonic and adult zebrafish (Danio rerio). Vis Neurosci 16:417-424

49. Otteson DC, D'Costa AR, Hitchcock PF (2001) Putative stem cells and the lineage of rod photoreceptors in the mature retina of the goldfish. Dev Biol 232:62-76

50. Cicero SA, Johnson D, Reyntjens S, Frase S, Connell S, Chow LM, Baker SJ, Sorrentino BP, Dyer MA (2009) Cells previously identified as retinal stem cells are pigmented ciliary epithelial cells. Proc Natl Acad Sci USA 106:6685-6690

51. Fischer AJ, Reh TA (2000) Identification of a proliferating marginal zone of retinal progenitors in postnatal chickens. Dev Biol 220:197-210

52. Haruta M, Kosaka M, Kanegae Y, Saito I, Inoue T, Kageyama R, Nishida A, Honda Y, Takahashi M (2001) Induction of photoreceptor-specific phenotypes in adult mammalian iris tissue. Nat Neurosci 4:1163-1164

53. Tropepe V, Coles BL, Chiasson BJ, Horsford DJ, Elia AJ, McInnes RR, van der Kooy D (2000) Retinal stem cells in the adult mammalian eye. Science 287:2032-2036

54. Otteson DC, Hitchcock PF (2003) Stem cells in the teleost retina: persistent neurogenesis and injury-induced regeneration. Vision Res 43:927-936

55. Bernardos RL, Barthel LK, Meyers JR, Raymond PA (2007) Late-stage neuronal progenitors in the retina are radial Muller glia that function as retinal stem cells. J Neurosci 27:70287040

56. Fausett BV, Gumerson JD, Goldman D (2008) The proneural basic helix-loop-helix gene asclla is required for retina regeneration. J Neurosci 28:1109-1117 
57. Fimbel SM, Montgomery JE, Burket CT, Hyde DR (2007) Regeneration of inner retinal neurons after intravitreal injection of ouabain in zebrafish. J Neurosci 27:1712-1724

58. Morris AC, Scholz TL, Brockerhoff SE, Fadool JM (2008) Genetic dissection reveals two separate pathways for rod and cone regeneration in the teleost retina. Dev Neurobiol 68:605619

59. Sherpa T, Fimbel SM, Mallory DE, Maaswinkel H, Spritzer SD, Sand JA, Li L, Hyde DR, Stenkamp DL (2008) Ganglion cell regeneration following whole-retina destruction in zebrafish. Dev Neurobiol 68:166-181

60. Thummel R, Kassen SC, Enright JM, Nelson CM, Montgomery JE, Hyde DR (2008) Characterization of Muller glia and neuronal progenitors during adult zebrafish retinal regeneration. Exp Eye Res 87:433-444

61. Yurco P, Cameron DA (2005) Responses of Muller glia to retinal injury in adult zebrafish. Vision Res 45:991-1002

62. Dyer MA, Cepko CL (2000) Control of Muller glial cell proliferation and activation following retinal injury. Nat Neurosci 3:873-880

63. Karl MO, Hayes S, Nelson BR, Tan K, Buckingham B, Reh TA (2008) Stimulation of neural regeneration in the mouse retina. Proc Natl Acad Sci USA 105:19508-19513

64. Bernardos RL, Raymond PA (2006) GFAP transgenic zebrafish. Gene Expr Patterns 6:1007-1013

65. Thummel R, Kassen SC, Montgomery JE, Enright JM, Hyde DR (2008) Inhibition of Muller glial cell division blocks regeneration of the light-damaged zebrafish retina. Dev Neurobiol 68:392-408

66. Raymond PA, Barthel LK, Bernardos RL, Perkowski JJ (2006) Molecular characterization of retinal stem cells and their niches in adult zebrafish. BMC Dev Biol 6:36

67. Thummel R, Enright JM, Kassen SC, Montgomery JE, Bailey TJ, Hyde DR (2010) Pax6a and Pax6b are required at different points in neuronal progenitor cell proliferation during zebrafish photoreceptor regeneration. Exp Eye Res 90:572-582

68. Hitchcock PF, Macdonald RE, VanDeRyt JT, Wilson SW (1996) Antibodies against Pax6 immunostain amacrine and ganglion cells and neuronal progenitors, but not rod precursors, in the normal and regenerating retina of the goldfish. J Neurobiol 29:399-413
69. MacLaren RE, Pearson RA, MacNeil A, Douglas RH, Salt TE, Akimoto M, Swaroop A, Sowden JC, Ali RR (2006) Retinal repair by transplantation of photoreceptor precursors. Nature 444:203-207

70. Otteson DC, Cirenza PF, Hitchcock PF (2002) Persistent neurogenesis in the teleost retina: evidence for regulation by the growth-hormone/insulin-like growth factor-I axis. Mech Dev 117:137-149

71. Bailey TJ, Fossum SL, Fimbel SM, Montgomery JE, Hyde DR (2010) The inhibitor of phagocytosis, O-phospho-L-serine, suppresses Muller glia proliferation and cone cell regeneration in the light-damaged zebrafish retina. Exp Eye Res. doi:10.1016/ j.exer.2010.07.017

72. Craig SE, Thummel R, Ahmed H, Vasta GR, Hyde DR, Hitchcock PF (2010) The zebrafish galectin Drgal1-12 is expressed by proliferating Muller glia and photoreceptor progenitors and regulates the regeneration of rod photoreceptors. Invest Ophthalmol Vis Sci 51:3244-3252

73. Kassen SC, Thummel R, Campochiaro LA, Harding MJ, Bennett NA, Hyde DR (2009) CNTF induces photoreceptor neuroprotection and Muller glial cell proliferation through two different signaling pathways in the adult zebrafish retina. Exp Eye Res 88:1051-1064

74. Taylor MR, Kikkawa S, Diez-Juan A, Ramamurthy V, Kawakami K, Carmeliet P, Brockerhoff SE (2005) The zebrafish pob gene encodes a novel protein required for survival of red cone photoreceptor cells. Genetics 170:263-273

75. Becker TS, Burgess SM, Amsterdam AH, Allende ML, Hopkins N (1998) not really finished is crucial for development of the zebrafish outer retina and encodes a transcription factor highly homologous to human Nuclear Respiratory Factor-1 and avian Initiation Binding Repressor. Development 125:4369-4378

76. Del Bene F, Wehman AM, Link BA, Baier H (2008) Regulation of neurogenesis by interkinetic nuclear migration through an apical-basal notch gradient. Cell 134:1055-1065

77. Starr CJ, Kappler JA, Chan DK, Kollmar R, Hudspeth AJ (2004) Mutation of the zebrafish choroideremia gene encoding Rab escort protein 1 devastates hair cells. Proc Natl Acad Sci USA 101:2572-2577 